Revista de Psicología Vol. 37 (1), 2019 (ISSN 0254-9247)

\title{
Participación social y política en Internet y brecha generacional ${ }^{1}$
}

\author{
Gisela Delfino $^{2}$, Maite Beramendi ${ }^{3}$, Elena Zubieta ${ }^{4}$ \\ Pontificia Universidad Católica Argentina², Universidad de Buenos Aires, \\ Argentina $^{3,4}$, CONICET $T^{2,4}$
}

\begin{abstract}
Los medios digitales posibilitan la aparición de distintas actividades que proveen de nuevas formas de participar en la vida social y política. Internet permite llegar a grupos que son normalmente inactivos o menos activos en las formas convencionales u offline de la participación política. Se buscó explorar la influencia que la frecuencia de conexión a Internet, la participación social Internet y la discusión política (hablar de política) tienen sobre la participación política en Internet en tres generaciones distintas $(n=502$, edad: 18-67). Los más jóvenes presentan mayor frecuencia de conexión y de realización de actividades en Internet mientras que el grupo generacional de mayor edad exhibe mayor frecuencia de discusión política. Tomadas en un nivel general las tres variables planteadas son relevantes para explicar actividad política en Internet, sin embargo, el peso de cada variable es distinto según la generación que se considere.

Palabras clave: activismo, brecha generacional, Internet, participación política, redes sociales.
\end{abstract}

\section{Social and political participation on the Internet and generational gap}

Digital media allows the emergence of different activities that provide new ways to participate in social and political life. The Internet allows reaching groups that are normally

1 Agradecimientos: Esta investigación se ha realizado bajo el financiamiento del proyecto PIP11220130100313CO (2014-2019) dirigido por la Prof. Dra. Elena Zubieta.

2 Doctora en Psicología, Universidad de Buenos Aires, Argentina. Profesora titular de la Pontificia Universidad Católica Argentina (UCA). Investigadora adjunta del Consejo Nacional de Investigaciones Científicas y Técnicas (CONICET) en el Centro de Investigaciones en Psicología y Psicopedagogía (CIPP) de la UCA. E-mail: gisela_delfino@uca.edu.ar ORCID: https://orcid.org/0000-0002-3732-184X

Doctora en Psicología, Universidad de Buenos Aires, Argentina. Ayudante de trabajos prácticos regular de la Universidad de Buenos Aires (UBA). E-mail: maite.beramendi@gmail. com ORCID: https://orcid.org/0000-0002-6113-5529

4 Doctora en Psicología Social, Universidad del País Vasco, España. Profesora adjunta regular de la Universidad de Buenos Aires (UBA). Investigadora independiente del Consejo Nacional de Investigaciones Científicas y Técnicas (CONICET) en la Facultad de Psicología de la UBA. E-mail: elenazubieta@hotmail.com ORCID: https://orcid.org/0000-0002-8789-737X 
inactive or less active in conventional or offline forms of political participation. We sought to explore the influence that frequency of Internet connection, Internet social participation and political discussion (talk about politics) have on political participation on the Internet in three different generations $(n=502$, age: 18-67). The youngest participants presented a higher frequency of connection and activities on the Internet, while the older generational group exhibited a greater frequency of political discussion. In general, the three variables are relevant to explain political activity on the Internet; however, the weight of each variable is different depending on the generation considered.

Keywords: activism, generation gap, Internet, political participation, social networks.

\section{Participaçáo social e política na Internet e brecha geracional}

A mídia digital permite o surgimento de diferentes atividades que proporcionam novas formas de participação na vida social e política. A Internet permite alcançar grupos que normalmente são inativos ou menos ativos em formas convencionais ou off-line de participação política. Procuramos explorar a influência que a frequência da conexão com a Internet, participação social na Internet e discussáo política (conversa sobre política) tem sobre a participação política na Internet em três diferentes geraçóes $(n=502$, idade: 18-67). Os mais jovens apresentam maior frequência de conexão e atividades na Internet, enquanto o grupo geracional mais antigo apresenta maior frequência de discussão política. A nível geral, as três variáveis são relevantes para explicar a atividade política na Internet, no entanto, o peso de cada variável é diferente dependendo da geração considerada.

Palavras-chave: ativismo, gap de geraçáo, Internet, participação política, redes sociais.

\section{Participation sociale et politique sur Internet et fossé générationnel}

Les médias digitaux permettent l'émergence de différentes activités qui offrent de nouvelles façons de participer à la vie sociale et politique. Internet permet d'atteindre des groupes normalement inactifs ou moins actifs dans des formes de participation politique conventionnelles ou hors ligne. Nous avons cherché à explorer l'influence de la fréquence des connexions Internet, de la participation sociale à Internet et des discussions politiques sur la participation politique sur Internet dans trois générations différentes $(n=502$, âge: 18-67). Les plus jeunes présentent une fréquence plus élevée de connexion et d'activités sur Internet, tandis que le groupe générationnel le plus âgé présente une fréquence plus grande de discussions politiques. Prises à un niveau général, les trois variables sont pertinentes pour expliquer l'activité politique sur Internet. Cependant, le poids de chaque variable est différent selon la génération considérée.

Mots-clés: activisme, écart de génération, Internet, participation politique, réseaux sociaux. 
El rápido crecimiento experimentado por la revolución de las nuevas tecnologías de información y, en especial, el uso de Internet a finales del siglo pasado ha permitido el surgimiento de nuevas formas de participación política (Valencia, 2011). Movilizaciones como la primavera árabe o el $15-\mathrm{M}$, cuyo objetivo central se relaciona con la defensa de la democracia, serían impensables sin comportamientos relacionados con el envío/respuesta de mensajes de texto, la utilización de foros de discusión o la distribución de información vía e-mail (Anduiza, Cristancho \& Sabucedo, 2014; Delfino \& Valencia, 2014). Las nuevas tecnologías han provocado una revolución en la forma de producir, consumir, construir la identidad y relacionarse (Anduiza, Contijoch, Gallego \& Salcedo, 2010). Un cambio de tal magnitud y profundidad como el que está produciendo la adopción de nuevas tecnologías sobre la economía, la sociedad o la cultura también afecta de manera significativa la relación de los ciudadanos con la política.

Sin embargo, para algunos autores (e.g., Halupka, 2016), la literatura existente sobre participación política tiene dificultad para comprender y explicar las nuevas formas de participación política, a menudo asociadas con la proliferación de la tecnología digital, que están cambiando la forma en que la gente ve y se involucra con la democracia. Internet se ha convertido en parte de la vida cotidiana de millones de personas. Según Villatoro y Silva (2005), Internet es bastante más que una plataforma tecnológica para el intercambio de información, consiste en una tecno-estructura cultural comunicativa, que permite la resignificación de las experiencias, del conocimiento y de las prácticas de interacción humana. No obstante, suelen plantearse dos posiciones contrapuestas en relación a las consecuencias de Internet (cf. Araya Dujisin, 2005; Colombo, 2006). Para algunos, constituye una herramienta al servicio de la homogeneización y hegemonía cultural que facilita la reproducción de las inequidades existentes 
(e.g. Bouza, 2003). Para otros, representa la promesa del bienestar en distintos ámbitos del desarrollo, como la educación, la superación de la pobreza, el mejoramiento de la gestión pública a través del gobierno, la promoción del capital social, la creación de ciudadanía, la protección de los derechos humanos y el fortalecimiento de la democracia (Porras, 2005).

El hecho de que la población esté dividida entre aquellos que acceden a esta herramienta y aquellos que no tienen esa posibilidad se conoce como brecha digital (DiMaggio \& Hargittai, 2001; Norris, 2001; van Dijk, 2006). La brecha digital tiene importantes implicaciones porque refleja y profundiza otro tipo de desigualdades socioeconómicas: el uso de Internet está fuertemente condicionado por variables como la edad o los estudios (van Deursen \& van Dijk, 2015a; Xenos \& Moy, 2007), y no se distribuye territorialmente de forma homogénea (Anduiza et al., 2010; Pew Research Center, 2015, Marzo). El acceso a las nuevas tecnologías aparece como un nuevo indicador de desigualdad (Schradie, 2011; van Deursen, van Dijk \& ten Klooster, 2015).

En este marco, son aún escasos en Latinoamérica los trabajos que permitan esclarecer qué impacto está teniendo el uso de Internet en el comportamiento político. El presente trabajo busca analizar las diferencias generacionales en la frecuencia de conexión a Internet, la participación social y política en Internet y la discusión política (hablar de política) en una muestra de residentes de la zona metropolitana de Buenos Aires. Asimismo, se propone analizar el poder explicativo de la frecuencia de conexión, la participación social en Internet y el hablar de política para predecir la participación política en Internet.

\section{Participación en Internet}

La conceptualización y la medición de la participación política ha sido un tema vibrantemente debatido en el último medio siglo (cf. Delfino \& Zubieta, 2010). La llegada de los medios digitales añade 
parámetros importantes para el debate y muestra la emergencia de formas de participación política que no estaban incluidas en las tipologías tradicionales (cf. Gil de Zuñiga, Veenstra, Vraga \& Shah, 2010). Según Theocharis (2015) los medios digitales posibilitan la aparición de nuevas y creativas formas de participar en la vida social y política. Un ejemplo de estas nuevas formas de participación política es el activismo informativo conceptualizado por Halupka (2016) al estudiar las protestas turcas. Los usuarios de Internet se dedican a la política en un nivel muy personal, renunciando a la acción colectiva centrada en la sociedad civil por un enfoque orientado al proyecto. La aparición de Internet exige una revisión del concepto de participación política por la razón evidente de que la gente puede ahora participar políticamente desde Internet.

Dos perspectivas se han planteado en el estudio de la relación entre la participación política y el uso de Internet: la optimista y la pesimista. Según la perspectiva optimista, el uso de Internet incrementa los niveles de participación política sugiriendo que Internet afecta positivamente la recogida de información política y las actividades políticas (Davis, 1999; Hill \& Hughes, 1998; Norris, 2001). Según la visión pesimista, Internet no tendría efecto en la participación política en general porque el mero uso no hace que las personas se interesen por la política (Bimber, 1999; Iyengar, 2001).

En la actualidad, respecto de la influencia de las nuevas tecnologías, Anduiza et al. (2010) organizan los planteos en tres perspectivas: (a) quienes sostienen que permiten un rápido acceso a la información política y a sus representantes, ayudando a un mayor acercamiento de la población a la política; (b) los que entienden que el uso de Internet deshumaniza, desvinculando a las personas de su entorno inmediato, llevando al desinterés por los asuntos públicos y a la conexión a la red orientada al entretenimiento; y (c) los que subrayan que la introducción de las nuevas tecnologías no produce cambios significativos en las opiniones y los comportamientos de los ciudadanos.

Estudios recientes han buscado aportar evidencia que ayude a esclarecer esta cuestión. Una nueva forma de participación ciudadana, 
y orientada al entretenimiento, se ha vehiculizado en Internet a partir de la red social Facebook. Un estudio realizado con jóvenes griegos halló que Facebook tiene consecuencias negativas sobre las formas tanto online como offline de participación política y cívica (Theocharis \& Lowe, 2015). Otros estudios (cf. Theocharis \& Quintelier, 2014) observaron relaciones positivas, aunque débiles o moderadas, entre la utilización de sitios de redes sociales y la participación cívica y política, concluyendo que la mayor equiparación en el acceso a Internet muestra un potencial genuino de participación política. Específicamente, Theocharis, Barberá, Fazekas y Popa (2015) señalan que Twitter es una plataforma de importante potencial para mejorar la disponibilidad de la información y hacer viables debates interactivos entre políticos y ciudadanos.

En esta misma línea, sobre la base de un estudio realizado en el Reino Unido, Gibson, Lusoli y Ward (2005) afirman que Internet está incrementando la cantidad de personas políticamente activas, concretamente en términos de llegar a grupos que son normalmente inactivos o menos activos en las formas convencionales u offline de la participación política. Sin embargo, no sería el tiempo invertido en Internet lo que llevaría a una propensión a participar en la vida pública, sino que habría que analizar otras variables como las actividades efectuadas en Internet (Quintelier \& Vissers, 2008). Según McDonald (2008), la frecuencia con que la gente, especialmente los más jóvenes, busca información política en Internet ha ido en aumento y continúa creciendo. La ciudadanía digital es más elevada entre los jóvenes. Los jóvenes, que suelen presentar niveles más bajos de participación cívica y política (Mossberger, Tolbert \& McNeal, 2008)., exhiben mayor probabilidad de buscar noticias políticas en Internet y en participar activamente en política a través de Internet. En este contexto, se plantea la primera hipótesis de investigación:

H1: La generación más joven, los menores de 34 ańos, presentan un mayor nivel de conexión y frecuencia de realización de actividades en internet, tanto sociales como política, que los adultos de mayor edad. 


\section{Discusión política}

El nivel de curiosidad que la política suscita en los ciudadanos, por ejemplo, hablar de política, permite distinguir entre sujetos políticamente activos y pasivos, y es central en los esquemas que unen los factores psicosociales a las actitudes y conductas políticas (van Deth, 1990). La discusión política, entendida como "examinar atentamente y particularmente una materia política con una o más personas" (Gil de Zuñiga, 2017, p. 66) contribuye a construcción de un sistema deliberativo. Discutir sobre política se asocia la confianza en las propias habilidades y suele predecir una mayor participación (Gil de Zúñiga, Barnidge \& Scherman, 2017; Gil de Zúñiga, Diehl \& Ardévol-Abreu, 2017). La discusión es una de los medios que los grupos tienen para tomar decisiones basadas en un razonamiento interpersonal y, en lo que hace al uso de Internet, la discusión y el intercambio de mensajes políticos es una forma de razonamiento que se da como resultado de la exposición a los medios (Ardèvol-Abreu, Diehl \& Gil de Zúñiga, 2017).

Campos (2011) explica que pueden distinguirse dos posturas en torno la discusión política en Internet: los que dicen que Internet fomenta la deliberación, el acercamiento y el interés, y los que consideran que no solo no promueve la participación, sino que además reduce el interés de los electores por los temas políticos manteniéndose la misma distancia que separaba a gobernantes de gobernados en el pasado. Xenos y Moy (2007) hallan que el uso de Internet tiene un efecto directo sobre la exposición y adquisición de información política pero también muestran que hay un patrón más complejo: en quienes presentan un mayor interés por la política el uso de Internet tiene un efecto mayor sobre la búsqueda y adquisición de información. Por su parte, Wang (2007) verifica, sobre la base de una muestra representativa de Taiwán, que el uso político de Internet fortalece el interés por la política y a sentimientos de confianza y eficacia que hacen a la persona más propensa a participar en campańas y en política. Un estudio desarrollado por el Centro de Investigaciones Sociológicas de España 
(Borge, Cardenal \& Malpica, 2012) encontró que el uso de Internet tiene un efecto positivo sobre la participación, independientemente del interés por la política, y que los usuarios de Internet que son habilidosos con este medio no necesitan estar motivados o interesados en la política para participar, al menos en una actividad política por Internet. Por su parte, Lupia y Philpot (2005) plantean que Internet no es un bloque con el que la gente interactúa en su conjunto, las personas interactúan con sitios web específicos y explican que el efecto de Internet sobre el interés por la política depende de los sitios web visitados, la edad y de cómo las personas evalúan ese sitio específico. En el contexto latinoamericano, Rottenbacher y Córdova (2014), en base a una muestra representativa de la ciudad de Lima, encontraron que la utilización de Internet, ya sea para discutir de política o participar políticamente, es aún incipiente. Para estos autores, no todas las personas se muestran dispuestas a deliberar políticamente en Internet. Aquellos con una orientación ideológica relacionada con la intolerancia a la ambigüedad y a la incertidumbre serían más renuentes a exponerse en un ambiente tan diverso como Internet. En este sentido, se propone que:

H2: La generación de mayor edad, quienes tienen más 50 ańos, presentan una mayor frecuencia de hablar de política que los que tienen menor edad.

Por último, y en lo que hace la posibilidad de explicar la participación política en Internet, se plantea el siguiente interrogante:

P1: Cuando se busca explicar la participación política online, ¡el poder predictivo de la frecuencia de conexión a internet, la actividad social online y el hablar de política es distinto según el grupo generacional que se considere?

\section{Método}

El diseño de la investigación fue transversal, retrospectivo ex-postfacto con un solo grupo y múltiples medidas (Montero \& León, 2007). Se trata de una encuesta analítica con una muestra no probabilística. 


\section{Participantes}

La muestra no probabilística intencional estuvo compuesta por 502 participantes residentes en la zona metropolitana de Buenos Aires. Un $51.4 \%$ eran mujeres y un $48.6 \%$ eran hombres. La media de edad fue de 34.03 ( $D E=15.29$ ), siendo el rango de edad 18-67 y la mediana igual a 25 ańos. En lo relativo a la autopercepción de nivel socioeconómico, en una escala de 1 (muy bajo) a 10 (muy alto), se obtuvo una media de 7.54 ( $D E=1.36)$, la mediana 8 y solo un $2.4 \%$ se identificó de nivel socioeconómico medio-bajo (marcó 4 o menos). Las generaciones se establecieron teniendo en cuenta lo planteado por Shah, Kwak y Holvert (2001) agrupándose a los participantes en tres categorías generacionales: millennial generation $(\mathrm{MG})$, generation $x(\mathrm{GX})$ y baby boom generation (BBG). La clasificación se efectuó utilizando la fecha de nacimiento según lo recomendado por el Pew Research Center (2015, Septiembre). Así, la MG quedó integrada por participantes con edades comprendidas entre 18 y 33 (291 participantes) al momento del relevamiento de datos (año 2015), la GX con edades entre 34 y 49 (77 participantes) y la BBG con edades entre 50 y 68 (134 participantes). La descompensación entre los grupos etarios se corresponde con la tasa de respuesta de cada una de las generaciones consideradas.

\section{Medición}

Se utilizó un cuestionario auto-administrado integrado por distintas preguntas y secciones de la cuales aquí solo se reportan las relativas a los objetivos planteados. Para medir la frecuencia de conexión a Internet se efectuaron tres preguntas: (a) ¿Cuántos días a la semana Ud. se conecta a Internet? Con un continuo de respuesta de $O$ días a 7 dias, (b) En un día típico, ¿cuántas horas está Ud. conectado a Internet? Para la cual el participante indicaba la cantidad de horas, y (c) En el transcurso de un día normal, ¿Ud. siempre está conectado a Internet? 
Con una opción de respuesta dicotómica: $0=$ no y $1=$ sí. Sobre la base de estas tres preguntas se calculó una nueva variable, conexión, que es el producto de darle a cada variable un peso igual a 1 y sumarlas (fórmula utilizada: valor en a / 7 + valor en b / 24 + valor en c). De esta forma, el índice de conexión a Internet oscila entre 0 y 3.

Para conocer las actividades realizadas en Internet, se les presentó una lista con diez actividades: cinco sociales y cinco políticas. El continuo de respuesta fue de $1=$ nunca a 5 = siempre. Las actividades sociales evaluadas son: (a) leer lo que postean otros, (b) postear sus comentarios, (c) mirar videos creados por otros, (d) seguir y compartir links de sitios de Internet en los medios sociales, y (e) compartir información no política en Internet (comercial, social). Las actividades políticas son: (a) firmar o compartir una petición en Internet, (b) compartir información política en Internet (noticias, fotos, videos, etc.), (c) poner "me gusta" o seguir temas o grupos políticos en los medios sociales, (d) unirse a un grupo político o a un grupo relacionado con una causa social en los medios sociales, y (e) crear un grupo político o relacionado con una causa social en los medios sociales. Sobre la base de estas actividades se calcularon dos variables: actividad social online y actividad politica. Ambas resultan del promedio de las cinco actividades sociales y políticas, respectivamente.

Por último, se preguntó ¿Con qué frecuencia $U d$. habla de politica con las siguientes personas? Las opciones eran: (a) familia, (b) amigos, (c) compañeros de trabajo, (d) vecinos, (e) personas que conoce por primera vez, y (f) personas de otro país. El continuo de respuesta se estableció entre 1 = nunca y 5 = siempre. Sobre la base de los seis ítems se calculó la variable hablar de politica (promedio de hablar de política).

\section{Procedimiento}

Para llegar a una muestra más amplia y heterogénea, se recolectaron los datos a través de Internet, enviando un e-mail a conocidos, que a su vez contactaron a otros conocidos (método bola de nieve), 
donde se proporcionaba un link desde el cual se accedía al cuestionario online. Se utilizó una plataforma de encuestas en línea, que fue programada para que no sea posible contestar más de una vez desde un mismo dispositivo (computadora, tableta, celular, etc.). El tiempo promedio necesario para completar el cuestionario fue de 30 minutos. La participación fue voluntaria y anónima, y las respuestas fueron recabadas durante el lapso de 25 días. Antes de comenzar con la encuesta, los participantes debían firmar un consentimiento informado, que cumple con los lineamientos para el comportamiento ético en las Ciencias Sociales y Humanas establecidos por el Consejo Nacional de Investigaciones Científicas y Técnicas (CONICET) de Argentina (Res. D. $\mathrm{N}^{\circ}$ 2857/06), en el que se les informaba acerca de los propósitos de la investigación.

\section{Análisis de datos}

Se efectuaron análisis descriptivos para cada variable analizada y se analizaron las correlaciones entre las mismas, tanto para toda la muestra como por generación. Para comparar las mismas variables según generaciones se efectuaron análisis de ANOVA de un factor. Se utilizó este estadístico paramétrico ya que, según la Teoría Central del Límite, se asume una distribución normal cuando el $\mathrm{n}$ es grande (Szretter-Noste, 2013). Finalmente, con el fin de conocer si el hablar de política, las actividades sociales online y la conexión a Internet predicen de igual forma las actividades políticas online en la muestra general y en cada una de las generaciones analizadas, se efectuaron análisis de regresión lineal múltiple de pasos sucesivos. Se trabajó con el paquete estadísticos SPSS v. 20.0 y para calcular el tamaño del efecto se utilizó el paquete estadístico G*Power v. 3.1.9.2 (Cárdenas \& Arancibia, 2014; Faul, Erdfelder, Buchner \& Lang, 2009). 


\section{Resultados}

Los participantes se conectan en promedio 6.46 días de los siete días de la semana $(D E=1.23)$ y el $79.1 \%$ declaró conectarse todos los días. En un día típico el tiempo medio de conexión es de 8.24 horas $(D E=5.65)$ y el $41.1 \%$ de la muestra declara estar 9 ó más horas al día conectado, explicando un $67.7 \%$ que en el transcurso de un día normal siempre está conectado. Las actividades online más realizadas son las sociales mientras que las actividades políticas presentan una baja frecuencia de realización, siendo las más habituales firmar una petición y poner "me gusta". Al comparar el promedio de realización de las actividades sociales con el de las actividades política, se obtiene que la diferencia entre las mismas es significativa $(t(501)=26.41 ; p<0.01$; IC95\% $[0.90,1.05] ; d=1.18)$. Por su parte, un $74,1 \%$ de los participantes dice no hablar nunca o pocas veces de política.

La Tabla 1 presenta los datos descriptivos para cada una de las escalas consideras y las correlaciones entre estas, tanto para toda la muestra general como para cada grupo etario. En lo que hace a la relación entre las variables, se observa una relación positiva y significativa entre, por un lado, la frecuencia de conexión a Internet y la realización de actividades sociales en Internet, y, por otro lado, la realización de actividades sociales en Internet y la realización de actividades políticas en Internet. También se detecta una relación positiva entre hablar de política y realizar actividades políticas en Internet, aunque esta asociación no es significativa para el caso de la GX.

\section{Diferencias por generación}

La Tabla 2 presenta los resultados obtenidos para la comparación según generación en las distintas variables. La GM exhibe un mayor promedio de conexión y realización de actividades sociales online que la GX y la BBG. En lo que hace a las actividades políticas online, la MG muestra una mayor realización que la GX. Mientras que la BBG habla más de política que la MG. 
Participación social y política en Internet y brecha generacional / Delfino, Beramendi, Zubieta

\section{Tabla 1}

Datos descriptivos y correlaciones entre las escalas

$M \quad D E \quad \alpha \quad$\begin{tabular}{c} 
Correlaciones \\
\cline { 3 - 5 }
\end{tabular}

Todas las generaciones $(\mathrm{n}=502$. Para conexión $\mathrm{n}=495)$

Conexión

$1.95 \quad .71 \quad---$

1

$.40^{* *}$

$.08 \quad-.06$

Actividad social

$2.76 \quad .88 \quad .80$

1

$.49^{* *}$

.01

Actividad política

$1.78 \quad .76 \quad .75$

1

$.42^{* *}$

Hablar de política

$2.43 \quad .70 \quad .78$

1

Millennial generation $(\mathrm{MG})(\mathrm{n}=291$. Para conexión $\mathrm{n}=289)$

Conexión

$2.13 \quad .61$

$---$

$1 \quad .27^{* *}$

.01

.04

Actividad social

$3.06 \quad .77 \quad .73$

1

$.44^{* *}$

.11

Actividad política

$\begin{array}{lll}1.86 & .78 \quad .74\end{array}$

1

$.47^{* *}$

Hablar de política

$2.33 \quad .70 \quad .78$

Generation $x(\mathrm{GX})(\mathrm{n}=77$. Para conexión $\mathrm{n}=75)$

Conexión

Actividad social

$1.82 \quad .75$

2.41

.82

.79

1

$.33^{* *}$

.08

Actividad política

1.55

.67

.78

1

$.47^{* *}$

.03

Hablar de política $\quad 2.40 \quad .71$

.83
1

Baby boom generation $(\mathrm{BBG})(\mathrm{n}=134$. Para conexión $\mathrm{n}=131)$

\begin{tabular}{lccccccc} 
Conexión & 1.64 & .75 & --- & 1 & $.39^{* *}$ & .12 & -.07 \\
Actividad social & 2.32 & .89 & .83 & & 1 & $.61^{* *}$ & .10 \\
Actividad política & 1.73 & .75 & .76 & & & 1 & $.50^{* *}$ \\
Hablar de política & 2.67 & .66 & .77 & & & & 1 \\
\hline
\end{tabular}

Nota. Para la variable Conexión no se reporta el $\alpha$ de Cronbach por tratarse de una escala construida en la base a la suma de 3 ítems con distinto nivel de medición. El coeficiente utilizado en las correlaciones es $r$ de Pearson y solo se muestran los resultados para la diagonal superior.

** $p<.01$. 


\section{Tabla 2}

Conexión, actividades online y hablar de politica según grupo generacional

\begin{tabular}{|c|c|c|c|c|}
\hline & \multicolumn{3}{|c|}{ Medias } & \multirow{2}{*}{$\mathrm{F}$} \\
\hline & $M G$ & $G X$ & $B B G$ & \\
\hline Conexión & 2.13 & 1.82 & 1.64 & $\begin{array}{l}\mathrm{F}(2,492)=25.90 ; p<.01 ; \mathrm{IC} 95 \%[1.51,2.02] ; \\
f=.78^{\mathrm{a}} .\end{array}$ \\
\hline Activ. sociales & 3.06 & 2.32 & 2.76 & $\begin{array}{l}\mathrm{F}(2,499)=46.08 ; p<.01 ; \mathrm{IC} 95 \%[2.68,2.84] ; \\
f=.35^{\mathrm{a}} .\end{array}$ \\
\hline Activ. políticas & 1.86 & 1.55 & 1.73 & $\begin{array}{l}\mathrm{F}(2,499)=5.49 ; p=.01 ; \mathrm{IC} 95 \%[1.71,1.85] ; \\
f=.15^{\mathrm{b}} .\end{array}$ \\
\hline $\begin{array}{l}\text { Hablar de } \\
\text { política }\end{array}$ & 2.33 & 2.40 & 2.67 & $\begin{array}{l}\mathrm{F}(2,499)=11.53 ; p<.01 ; \mathrm{IC} 95 \%[2.37,2.49] ; \\
f=.28^{\mathrm{b}}\end{array}$ \\
\hline
\end{tabular}

${ }^{\text {a }}$ Prueba post-hoc T3 de Dunnett. ${ }^{\text {b }}$ Prueba post-hoc Scheffé.

\section{Determinantes de la actividad política online}

Con el fin de establecer el poder explicativo que la frecuencia de conexión a Internet, la actividad social online y el hablar de política pueden tener sobre la actividad política online se procedió a efectuar una regresión lineal múltiple por pasos sucesivos tanto con la muestra total como con cada una de las generaciones (ver Tabla 3).

\section{Tabla 3}

Regresión lineal para actividad politica online

\begin{tabular}{lcccc}
\hline & Toda la muestra $^{1}$ & $\mathrm{GM}^{2}$ & $\mathrm{GX}^{3}$ & $\mathrm{BBG}^{4}$ \\
\hline Conexión & $-.11^{* *}$ & $-.13^{* *}$ & Excluida & Excluida \\
Activ. sociales & $.53^{* * *}$ & $.43^{* * *}$ & $.49^{* * *}$ & $.56^{* * *}$ \\
Hablar de política & $.40^{* * *}$ & $.43^{* * *}$ & Excluida & $.45^{* * *}$ \\
\hline$R^{2}$ corregida & .42 & .39 & .23 & .56 \\
\hline
\end{tabular}

Nota. Coeficientes estandarizados $(\beta) .{ }^{1} \mathrm{n}=497 . F(3,491)=118.52 ; p<.01 ; f^{2}=.72 .{ }^{2} \mathrm{n}=289$. $F(3,285)=61.39 ; p<.01 ; f^{2}=.65 .{ }^{3} \mathrm{n}=75 . F(1,73)=23.21 ; p<.01 ; f^{2}=.32 .{ }^{4} \mathrm{n}=131$. $F(2,128)=84.55 ; p<.01 ; f^{2}=1.32 .{ }^{* *} \mathrm{p}<.01,{ }^{* * *} \mathrm{p}<.001$. 
Se encontró que las tres variables explican la actividad política online en la GM. Sin embargo, para las otras dos generaciones, la frecuencia de conexión a internet resulta no significativa y el hablar de política no es significativo para la GX.

\section{Discusión}

Los participantes de este estudio presentan una alta frecuencia de conexión a Internet, posiblemente mayor que la del promedio de la población de la región. Se trata de usuarios de Internet que contestaron el cuestionario por esa vía. Asimismo, es importante tener en cuenta que los mismos se identificaron como de nivel socioeconómico medio-alto y se sabe que mayores ingresos se asocian a un mayor uso de Internet (Anduiza et al., 2010; van Deursen et al., 2015). Esta preferencia por Internet - comprendida a través de la cantidad de tiempo que están conectados-, corrobora lo hallado por el Pew Research Center (2015, Marzo) que señala que "una vez conectados, los usuarios de los países emergentes y en desarrollo adoptan las redes sociales como actividad digital preferida" (p. 6).

Las diferencias entre las tres generaciones analizadas son claras, los menores de 34 años presentan una frecuencia de conexión mayor, al tiempo que también realizan más actividades sociales y políticas online, y menor frecuencia de hablar de política. Estudios previos señalaban una mayor utilización de Internet entre los más jóvenes, especialmente atribuida a mayores habilidades (van Deursen \& van Dijk, 2015a). No obstante, Anduiza et al. (2010), al trabajar solo con usuarios de Internet, verificaban una correlación positiva entre edad y realización de actividades políticas online que los resultados aquí analizados contradicen.

Van Dijk (2006) y van Deursen \& van Dijk (2015b) plantean que para analizar la brecha digital es necesario tener en cuenta cuatro niveles de acceso: (a) la motivación, el interés, (b) el acceso físico, conectarse, (c) las habilidades, y (d) el uso. En términos de conexión, es decir con qué frecuencia se conectan, la MG presenta mayor utilización que las 
otras generaciones. Manteniéndose esta tendencia al considerar actividades, tanto sociales como políticas, realizadas en Internet. De forma contraria, al considerar la frecuencia de discusión política, operacionalizada a partir de la frecuencia con que los participantes declaran hablar de política, es la generación de mayor edad, la BBG, es la que presenta mayores índices.

A la hora predecir la participación política online, el análisis en función de las generaciones señala su relevancia en los estudios sobre el uso de Internet y la participación política. Si bien para las tres generaciones el efectuar actividades sociales en Internet resulta central, el hablar de política, en tanto intercambio de opiniones o puntos de vista con otros, no parece ser relevante, especialmente para la GX. Asimismo, la frecuencia de discusión política resulta ser la única variable de las tres consideradas que explica la participación política en Internet cuando se considera a la generación de mayor edad, la BBG.

Queda pendiente para futuras investigaciones abordar la pregunta relativa a si existe relación entre la participación política online y la participación política offline, y si la hay, cuál es el sentido de esta relación. ¿Podrá la participación política online favorecer el interés e incentivar la participación política offline? El estudio de Rottenbacher y Córdova (2014) demuestra que el discutir de política offline favorece el intercambio de opiniones online y no al revés. Nuevos estudios deberán analizar si existe algún tipo de relación causal entre ambos tipos de deliberación y participación política.

Con frecuencia la baja participación de los jóvenes en política convencional se interpreta como un indicador de menor compromiso cívico (cf. Varela, Martínez \& Cumsille, 2015). Sin embargo, los resultados aquí analizados indican que si bien ese menor interés relativo por los temas políticos (hablan menos de política) puede ser cierto, la MG si realiza actividades políticas en Internet. Las nuevas tecnologías, e Internet en particular, son relevantes a la hora de considerar la participación política de las nuevas generaciones.

Con relación a las limitaciones, la muestra de este estudio es intencional y solo ha incluido participantes de la zona Metropolitana. 
Asimismo, es importante señalar que los grupos generacionales tienen distinta cantidad de participantes. Para próximas investigaciones se propone, además de balancear la proporción de participantes en cada generación, ampliar la muestra a otros territorios del país y comprobar la brecha digital y su relación con la participación política a partir de la región geográfica. También sería deseable incorporar en el estudio participantes con distintos niveles socioeconómicos.

\section{Referencias}

Anduiza, E., Contijoch, M., Gallego, A. \& Salcedo, J. (2010). Internet y participación política en España. Madrid: Centro de Investigaciones Sociológicas. Recuperado de http://libreria.cis.es/static/ pdf/OyA63a.pdf

Anduiza, E., Cristancho, C. \& Sabucedo, J. M. (2014). Mobilization through online social networks: the political protest of the Indignados in Spain. Information, Communication \& Society, 17(6), 750-764. https://doi.org/10.1080/1369118X.2013.808360

Araya Dujisin, R. (2005). Internet, política y ciudadanía. Nueva Sociedad, 195, 56-71. Recuperado de http://biblioteca2012. hegoa.efaber.net/system/ebooks/14976/original/Gobierno_ Electronico_y_Democracia.pdf

Ardèvol-Abreu, A., Diehl, T. \& Gil de Zúñiga, H. (2017). Antecedents of internal political efficacy incidental news exposure online and the mediating role of political discussion. Politics, 1-19. https:// doi.org/10.1177/0263395717693251

Bimber, B. (1999). The Internet and citizen communication with government: does the medium matter? Political Communication, 16(4), 409-428. https://doi.org/10.1080/105846099198569

Borge, R., Cardenal, A. S. \& Malpica, C. (2012). El impacto de Internet en la participación política: revisando el papel del interés político. ARBOR Ciencia, Pensamiento y Cultura, 188(756), 733-750. https://doi.org/10.3989/arbor.2012.756n4008 
Bouza, F. (2003). Tendencias a la desigualdad en Internet: la brecha digital (digital divide) en España. En J. Tezanos, J. Tortosa \& A. Alaminos (Eds.). Tendencias en desvertebración social y en politicas de solidaridad (pp. 93-121). Madrid: Sistema.

Campos, E. (2011). La ciberdemocracia en el Congreso de los Diputados (2004-2008). Madrid: Congreso de los Diputados.

Cárdenas, M. \& Arancibia, H. (2014). Potencia estadística y cálculo del tamaño del efecto en G*Power: complementos a la prueba de significación estadística y su aplicación en psicología. Salud \& Sociedad, 5(2), 210-224. https://doi.org/10.22199/S07187475. 2014.0002 .00006

Colombo, C. (2006). Innovación democrática y TIC, ¿̨hacia una democracia participativa? Revista de Internet, Derecho y Politica, 3, 28-40. Recuperado de http://www.uoc.edu/idp/3/dt/esp/ colombo.pdf

Davis, R. (1999). The web of politics. The Internet's impact on the American political system. Nueva York: Oxford University Press.

Delfino, G. \& Valencia, J. F. (2014). Participación política. En E. Zubieta, J. F. Valencia \& G. Delfino (Eds.), Psicología social y política: procesos teóricos y estudios aplicados (pp. 255-295). Buenos Aires: Eudeba.

Delfino, G. \& Zubieta, E. (2010). Participación política: concepto y modalidades. Anuario de Investigaciones, 17, 211-220.

DiMaggio, P. \& Hargittai, E. (2001). From the 'Digital Divide' to 'Digital Inequality': Studying Internet Use as Penetration Increases. Working Paper \#15, Princeton University. Recuperado de http://www.princeton.edu/ -artspol/workpap/WP15\%20-\% 20DiMaggio+Hargittai.pdf

Faul, F., Erdfelder, E., Buchner, A. \& Lang, A.-G. (2009). Statistical power analyses using $\mathrm{G}^{*}$ Power 3.1: Tests for correlation and regression analyses. Behavior Research Methods, 41(4), 11491160. https://doi.org/10.3758/BRM.41.4.1149

Gibson, R. K., Lusoli, W. \& Ward, S. (2005). Online participation in the UK: testing a 'contextualised' model of Internet effects. 
British Journal of Politics and International Relations, 7(4), 561583. https://doi.org/10.1111/j.1467-856x.2005.00209.x

Gil de Zúníga, H. (2017). Los atributos de la discusión política interpersonal como antecedentes de la elaboración cognitiva. Revista Española de Investigaciones Sociológicas, 157, 65-84. https://doi. org/10.5477/cis/reis.157.65

Gil de Zúñiga, H., Barnidge, M. \& Scherman, A. (2017). Social media social capital, offline social capital, and citizenship: Exploring asymmetrical social capital effects. Political Communication, 34(1), 44-68. https://doi.org/10.1080/10584609.2016.1227000 Gil de Zúńiga, H., Diehl T. \& Ardévol-Abreu, A. (2017). Internal, external, and government political efficacy: effects on news use, discussion, and political participation. Journal of Broadcasting \& Electronic Media, 61(3), 574-596. https://doi.org/10.1080/088 38151.2017 .1344672

Gil de Zúńiga, H., Veenstra, A., Vraga, E. \& Shah, D. (2010). Digital democracy: Reimagining pathways to political participation. Journal of Information Technology \& Politics, 7(1), 36-51. https:// doi.org/10.1080/19331680903316742

Halupka, M. (2016). The rise of information activism: how to bridge dualisms and reconceptualise political participation. Information, Communication \& Society. Publicado online, 10 de enero, 1-17. https://doi.org/10.1080/1369118X.2015.1119872

Hill, K. A. \& Hughes, J. E. (1998). Cyberpolitics: citizen activism in the age of the Internet. Lanham, Md.: Rowman and Littlefield.

Iyengar, J. (2001). The method is the message. Political Communication, 18(2), 225-229. https://doi.org/10.1080/105846001750322998 Lupia, A. \& Philpot, T. S. (2005). Views from inside the net: how websites affect young adults' political interest. The Journal of Politics, 67(4), 1122-1142. https://doi.org/10.1111/j.1468-2508.2005.00353.x McDonald, J. (2008). The benefits of society online: civic engagement. En K. Mossberger, C. J. Tolbert \& R. S. McNeal, Digital citizenship: The Internet, society, and participation (pp. 47-66). Cambridge: MIT Press. 
Montero, I. \& León, O. G. (2007). A guide for naming research studies in Psychology. International Journal of Clinical and Health Psychology, 7(3), 847-862. Recuperado de http://www.aepc.es/ ijchp/articulos_pdf/ijchp-256.pdf

Mossberger, K., Tolbert, C. J. \& McNeal, R. S. (2008). Digital citizenship: The Internet, society, and participation. Cambridge: MIT Press.

Norris, P. (2001). Digital divide: civic engagement, information poverty, and the Internet worldwide. Cambridge: Cambridge University Press. https://doi.org/10.1017/CBO9781139164887

Pew Research Center (2015, Marzo). Internet seen as positive influence on education but negative influence on morality in emerging and developing nations. Recuperado de http://www.pewglobal.org/ files/2015/03/Pew-Research-Center-Technology-ReportFINAL-March-19-20151.pdf

Pew Research Center (2015, Septiembre). The whys and hows of generations research. Recuperado de http://www.people-press.org/2015/09/03/ the-whys-and-hows-of-generations-research/generations_2/

Porras, J. I. (2005). Internet y las nuevas oportunidades para la deliberación pública en los espacios locales. Nueva Sociedad, 195, 56-71. Recuperado de http://biblioteca2012.hegoa.efaber. net/system/ebooks/14976/original/Gobierno_Electronico_y_ Democracia.pdf

Quintelier, E. \& Vissers, S. (2008). The effect of Internet use on political participation. An analysis of survey results for 16-year-olds in Belgium. Social Science Computer Review, 26(4), 411-427. https://doi.org/10.1177/0894439307312631

Rottenbacher, J. M. \& Córdova, C. (2014). El Autoritarismo y la justificación de la inequidad como condicionantes ideológicos de los niveles de deliberación y participación política. Avances en Psicologia Latinoamericana, 32(3), 495-513. https://doi.org/ 10.12804/apl32.03.2014.10

Schradie, J. (2011). The digital production gap: the digital divide and Web 2.0 collide. Poetics, 39(2), 145-168. https://doi.org/ 10.1016/j.poetic.2011.02.003 
Shah, D. V., Kwak, N. \& Holvert, R. L. (2001). "Connecting” and "disconnecting" with civic life: patterns of Internet use and production of social capital. Political Communication, 18(2), 141-162. https://doi.org/10.1080/105846001750322952

Szretter-Noste, E. (2013). Apunte de regresión lineal. Buenos Aires: Universidad de Buenos Aires. Recuperado de http://mate. dm.uba.ar/ meszre/apunte_regresion_lineal_szretter.pdf

Theocharis, Y. \& Lowe, W. (2015). Does Facebook increase political participation? Evidence from a field experiment. Information, Communication \& Society. Publicado online, 17 de Diciembre, 1-32. https://doi.org/10.1080/1369118X.2015.1119871

Theocharis, Y. \& Quintelier, E. (2014). Stimulating citizenship or expanding entertainment? The effect of Facebook on adolescent participation. New Media \& Society. Publicado online, 27 de Agosto, 1-20. https://doi.org/10.1177/1461444814549006

Theocharis, Y. (2015). The Conceptualization of Digitally Networked Participation. Social Media + Society, 1(2), 1-14. https://doi. org/10.1177/2056305115610140

Theocharis, Y., Barberá, P., Fazekas, Z. \& Popa, S. A. (2015, Septiembre). $A$ bad workman blames his tweets. The consequences of citizens uncivil twitter use when interacting with party candidates. 2015 Annual Meeting of the American Political Science Association, San Francisco, CA.

Valencia, J. F. (2011). Mobilization: Peaceful and Violent. En D. Christie (Ed.), The Encyclopedia of Peace Psychology (pp. 161). Hoboken, NJ: Wiley. https://doi.org/10.1002/9780470672532. wbepp 161

van Deth, J. W. (1990). Interest in politics. En M. K. Jennings, J. W. van Deth, S. H. Barnes, D. Fuchs, F. L. Heunks, R. F. Inglehart,... J. J. A. Thomassen (Eds.), Continuities in political action: a longitudinal study of political orientations in three Western democracies (pp. 275-312). Berlin- New York: De Gruyter and Aldine. van Deursen, A. \& van Dijk, J. (2015a). Internet skill levels increase, but gaps widen: a longitudinal cross-sectional analysis (2010-2013) 
among the Dutch population. Information, Communication \& Society, 18(7), 782-797. https://doi.org/10.1080/1369118X. 2014.994544

van Deursen, A. \& van Dijk, J. (2015b). Toward a multifaceted model of Internet access for understanding digital divides: an empirical investigation. The Information Society, 31(5), 379-391. https:// doi.org/10.1080/01972243.2015.1069770

van Deursen, A., van Dijk, J. \& ten Klooster, P. M. (2015). Increasing inequalities in what we do online: a longitudinal cross sectional analysis of Internet activities among the Dutch population (2010 to 2013) over gender, age, education, and income. Telematics and Informatics, 32(2), 259-272. https://doi.org/10.1016/j. tele.2014.09.003

van Dijk, J. (2006). Digital divide research, achievements and shortcomings. Poetics, 34(4-5), 221-235. https://doi.org/10.1016/j. poetic. 2006.05 .004

Varela, E., Martínez, M. L. \& Cumsille, P. (2015). ¿Es la participación política convencional un indicador del compromiso cívico de los jóvenes? Universitas Psychologica, 14(2), 715-730. https:// doi.org/10.11144/Javeriana.upsy14-2.eppc

Villatoro, P. \& Silva, A. (2005). Estrategias, programas y experiencias de superación de la brecha digital y universalización del acceso a las nuevas tecnologias de información y comunicación (TIC). Un panorama regional. Santiago de Chile: CEPAL, Naciones Unidas.

Wang, S.-I. (2007). Political use of the Internet, political attitudes and political participation. Asian Journal of Communication, 17(4), 381-395. https://doi.org/10.1080/01292980701636993

Xenos, M. \& Moy, P. (2007). Direct and differential effects of the Internet on political and civic engagement. Journal of Communication, 57(4), 704-718. https://doi.org/10.1111/j.1460-2466. 2007.00364.x

Recibido: 10 de noviembre, 2017

Revisado: 31 de mayo, 2018 Aceptado: 3 de setiembre, 2018 